\section{NÍVEIS DE ESTRESSE E VARIABILIDADE DA FREQUÊNCIA CARDÍACA EM PROFESSORES UNIVERSITÁRIOS}

Ronaldo Henrique Cruvinel Júnior ${ }^{1}$

Graduando em Fisioterapia, Centro Universitário de Formiga (UNIFORMG), E-mail:

ronaldocruvinel1996@hotmail.com

\section{Ana Paula de Lourdes} Pfister $^{2}$

Fisioterapeuta Mestre em Promoção da Saúde, Centro Universitário de Formiga (UNIFOR-MG), E-mail: aplp29@hotmail.com

\section{Elaine Karla Ferreira ${ }^{3}$,}

Graduanda em Fisioterapia, Centro Universitário de Formiga (UNIFORMG), E-mail:

nanyk_001@hotmail.com

Jayne Cristina Guimarães ${ }^{4}$ Graduanda em Enfermagem, Centro Universitário de Formiga (UNIFOR$M G), E-m a i l:$

jaynecguimaraes08@gmail.com

\author{
LEVELS OF STRESS AND HEART RATE VARIABILITY IN \\ UNIVERSITY TEACHERS
}

\section{RESUMO}

O professor universitário é altamente exigido tanto fisicamente, quanto mentalmente perante a realização de sua docência, estes podem apresentar estresse que é um dos principais causadores de alteração do funcionamento do sistema nervoso autônomo, sendo assim a variabilidade da frequência cardíaca (VFC) constitui uma ferramenta que permite a quantificação destas alterações, através de uma análise não invasiva do tônus vagal e simpático. Tendo como objetivo: Avaliar a modulação autonômica cardíaca a partir da variabilidade da frequência cardíaca em professores universitários de uma instituição de Ensino Superior do Centro Oeste Mineiro. Materiais e Métodos: Foram avaliados 30 professores universitários, de ambos os gêneros, com idade entre 30 e 59, foram captados os intervalos $\mathrm{R}$ - $\mathrm{R}$ de todos os voluntários durante cinco minutos em repouso utilizando um cardiofrequencímetro, e posteriormente os dados foram enviados ao software Kubios HRV Standard 3.0, para realização das análises. Resultados: Quando comparado os valores médios dos índices LF, HF, RMSSD e pNN50\% entre os professores universitários que não apresentavam estresse e aqueles em fase de resistência, ficou evidenciado que não houve diferença estatística significativa. As variáveis nível de atividade física, Índice de Massa Corporal e tempo de docência, não apresentaram correlação linear estatisticamente significativa com os índices de VFC. Conclusão: Os achados do presente estudo mostram que baixos níveis de estresse não alteram a modulação autonômica cardíaca destes indivíduos, não predispondo a patologias cardiovasculares. Não houve ainda influência do nível de atividade física, IMC e tempo de docência sobre os índices de VFC.

PALAVRAS-CHAVE: Estresse Ocupacional, Sistema Nervoso Autônomo, Docentes. 


\section{ABSTRACT}

The university teacher is highly required both physically and mentally in the performance of his teaching, these can present stress that is one of the main causes of altered autonomic nervous system functioning, thus heart rate variability (HRV) constitutes a tool that allows the quantification of these changes through a non-invasive analysis of the vagal and sympathetic tonus. Objective: To evaluate the cardiac autonomic modulation based on the heart rate variability in university professors of a Higher Education Institution of the Center Oeste Mineiro. Materials and methods: 30 university teachers of both genders, aged between 30 and 59, were assessed the RR intervals of all volunteers during five minutes at rest using a cardiofrequency meter, and later the data were sent to Kubios HRV Standard 3.0 software, to carry out the analyzes. Results: When comparing the mean values of the LF, HF, RMSSD and pNN50\% indices among university teachers who did not present stress and those in the resistance phase, it was evidenced that there was no significant statistical difference. The variables physical activity level, body mass index and teaching time did not present a statistically significant correlation with HRV indices. Conclusion: The present study findings show that low levels of stress do not alter the autonomic cardiac modulation of these individuals, not predisposing to cardiovascular pathologies. There was still no influence of physical activity level, BMI and teaching time on the HRV indexes.

KEYWORDS: Occupational stress. Autonomic Nervous System. Teachers.

\section{INTRODUÇÃO}

O ser humano está sujeito a diversas mudanças adaptativas durante a vida, requerendo assim uma capacidade de adaptação desenvolvida, o que envolve mobilização de energia, tanto no aspecto físico quanto mental. O desequilíbrio entre a velocidade de demanda adaptativa e a capacidade em se adaptar, leva a uma possível situação de conflito no organismo, desencadeando situações de estresse, através das quais esgotam os recursos de que se dispõem os indivíduos frente a uma situação ameaçadora, pode ser caracterizado ainda por um conjunto de condições bioquímicas do organismo humano que refletem a tentativa do corpo de realizar os ajustes de acordo com as exigências do ambiente. (GOULART JUNIOR; LIPP, 2008).

As alterações descritas acima quando desencadeadas no ambiente de trabalho são referidas através do termo estresse ocupacional, sendo este um fator externo causador de estresse nos indivíduos (DE SOUZA et al, 2016).

O estresse é um processo fisiológico do organismo dos seres humanos, age como desencadeador da motivação contribui para melhora do desempenho de atividades e age como estimulante para superar desafios, este estresse fisiológico é intitulado como eustress. Porém, o excesso na liberação de estresse pode levar a ocorrências patológicas no organismo humano, este é chamado de distress, que é anormal (MARTINS, TOURINHO, SANTOS, 2016).

Em 2013, a Organização Mundial da Saúde (OMS), alertou que a partir de 2030 o estresse juntamente com a depressão se tornará a principal causa de morte no mundo, podendo assim ser considerado uma epidemia.

Servilha(2012), afirma que o professor universitário é altamente exigido tanto fisicamente, quanto mentalmente durante a realização de sua docência. $O$ excesso de atividades realizadas por professores universitários, dentre outras 
condições como: poucas horas de sono, sedentarismo e falta de lazer, criam condições que favorecem o aparecimento do estresse, o que leva a um possível comprometimento de sua saúde.

O estresse pode causar uma série de alterações prejudiciais ao organismo humano, tais como: hipervigilância, pesadelos, medo, ansiedade, dificuldade de concentração, mudanças de humor, memória prejudicada e diminuição do rendimento na realização de atividades, sintomas estes repercutem gravemente no desempenho profissional de qualquer indivíduo, em especial aqueles que trabalham na docência e atividades afins, e que dependem de um bom rendimento intelectual. (FERREIRA et al, 2016).

Rodrigues et al (2016) afirmam que o estresse é um dos principais causadores de alteração do funcionamento do sistema nervoso autônomo, sendo assim a variabilidade da frequência cardíaca constitui uma ferramenta que permite a quantificação destas alterações, através de uma análise não invasiva do tônus vagal e simpático.

A variabilidade da frequência cardíaca, representa a variação entre os intervalos $\mathrm{R}$-R de batimentos cardíacos, apontando assim o balanço autonômico entre o sistema simpático e parassimpático. Alta variabilidade da frequência cardíaca representa uma boa adaptação cardíaca, do contrário a baixa variabilidade da frequência cardíaca é indicativo de uma insuficiência na modulação autonômica cardíaca, sendo que esta redução pode ser relacionada a estados patológicos e modificações fisiológicas tais como o estresse mental. (OLIVEIRA-SILVA et al, 2016).

Sendo assim, a variabilidade da frequência cardíaca tornou-se uma importante ferramenta de avaliação do funcionamento do sistema nervoso autônomo, o qual tem grande importância no equilíbrio homeostático do organismo humano, é utilizada como preditora de funções internas do organismo, tanto em estados fisiológicos quanto patológicos, sendo bastante eficaz para avaliar e identificar comprometimentos na saúde. (LONGHI, 2009).

Partindo desse pressuposto o presente estudo procurou avaliar a modulação autonômica cardíaca a partir da variabilidade da frequência cardíaca em professores universitários de uma instituição de Ensino Superior do Centro Oeste Mineiro.

\section{METODOLOGIA}

Trata-se de um estudo do tipo Observacional transversal com abordagem quantitativa.

A presente pesquisa somente foi iniciada após a aprovação do Comitê de Ética do Centro Universitário de Formiga (UNIFOR-MG). O protocolo de pesquisa foi inscrito na Plataforma Brasil sobre o número CAAE 73277517.1.0000.5113 no ano de 2017. Após o parecer favorável do Comitê de Ética por meio do parecer de número 2.290.622, foi solicitado aos interessados em participar da pesquisa que assinassem em duas vias o termo de consentimento livre e esclarecido (TCLE). Estas e demais considerações éticas se basearam na resolução 466/12 do Conselho Nacional de Saúde (CNS).

\section{AMOSTRA:}

A amostra foi obtida por conveniência, sendo selecionados 65 professores universitários, destes trinta e cinco $(53,8 \%)$ foram excluídos, cinco $(7,7 \%)$ por apresentarem patologias que interferem nos índices de variabilidade da 
frequência cardíaca, como: doença cardíaca, insuficiência renal ou hepática, doença pulmonar obstrutiva crônica, doenças autoimunes, Diabetes Mellitus, tireoidopatias e portadores de marca-passo, e trinta (46,1\%) foram excluídos por não dispor de tempo para participarem da pesquisa.

Foram considerados para este estudo 30 professores universitários de ambos os gêneros e com idade entre 30 e 59 anos.

\section{INSTRUMENTAÇÃO E COLETA DE DADOS:}

O presente estudo foi realizado no Centro Universitário de Formiga - MG, sendo que a coleta de dados foi realizada em uma sala de avaliação da Clínica Escola de Saúde. Os participantes foram orientados a não consumirem bebidas contendo cafeína, e bebidas alcoólicas nas 24 horas que antecederem a avaliação, sendo que estas interferem na mensuração dos dados da VFC. A coleta de dados foi toda realizada no período noturno das 18:00 ás 18:30 visando anular possíveis alterações hormonais decorrentes do ciclo circadiano. Foi aplicado o termo de consentimento livre e esclarecido - TCLE, com intuito de obter a assinatura do voluntário que deseja fazer parte do projeto. Foi utilizado uma Ficha de dados sociodemográficos para registrar as variáveis: sociodemográficas, laborais, clínicas e os critérios de exclusão do estudo.

A Aferição dos dados vitais foi feita após o voluntário permanecer por cinco minutos em repouso, em seguida as medidas antropométricas foram obtidas com o indivíduo descalço, na posição ereta; o peso foi avaliado em balança da marca G.Tech®, previamente calibrada e a estatura verificada em fita métrica da marca MacroLife®. O índice de massa corporal (IMC) foi obtido dividindo-se o peso em quilogramas pelo quadrado da estatura em metros.

A avaliação do nível de estresse foi feita através da aplicação questionário: Inventário de Sintomas de Estresse para Adultos (ISSL) validado por Lipp e Guevara em 1994, o qual permite identificar a presença e a fase de estresse em que o indivíduo se encontra (alerta, resistência ou exaustão).

Para realizar a análise da Variabilidade da Frequência Cardíaca (VFC) os voluntários permaneceram em repouso durante cinco minutos para que fosse atingido os valores basais de frequência cardíaca, logo em seguida foi realizado o registro da VFC durante um período de cinco minutos, permanecendo os voluntários em posição sentada durante todo o período de coleta. A fita torácica do cardiofrequencímetro foi retirada cinco minutos após o registro ter sido efetuado, para evitar possíveis alterações de sinal e captação de artefatos. Para o registro foram captados os intervalos $R-R$ sucessivos no domínio da frequência e do tempo, utilizando o cardiofrequencímetro V800 da marca Polar® por meio de um cinto elástico fixado no terço inferior do esterno.

Os intervalos R-R de cada um dos voluntários foram gravados, e em seguida analisados utilizando o software Kubios HRV Standard 3.0, para obtenção dos índices da Variabilidade da Frequência Cardíaca (VFC). Estes índices são cálculos matemáticos que expressam a VFC, sendo estes gerados de forma automática pelo software Kubios HRV Standard 3.0.

Os índices utilizados para análise foram: RMSSD: Representa a raiz quadrada da média dos quadrados das diferenças de sucessivos intervalos R-R. Para ser mais fácil, é a média das diferenças entre RR adjacente. Fisiologicamente, RMSSD nos diz sobre as mudanças de intervalos RR consecutivos. Esse parâmetro reflete principalmente a atividade do sistema nervoso parassimpático. pNN50: Porcentagem das diferenças sucessivas entre os intervalos RR que são maiores que $50 \mathrm{~ms}$. Esse parâmetro reflete principalmente a atividade do 
sistema nervoso parassimpático. HF: Alta Frequência durante um intervalo de 0,15-0,40 Hz. Relaciona-se com frequência respiratória e atividade vagal. LF: Baixa frequência correspondente ao intervalo de 0,04 a $0.15 \mathrm{~Hz}$. Sistema Nervoso Simpático e Parassimpático podem estar envolvidos, porém para muitos autores é considerado marcador de ativação simpática e reflete também a função dos barorreceptores. (MARÃES, 2010; CAMM et al, 1996)

\section{ANÁLISE DE DADOS:}

Os dados obtidos foram tabulados na planilha do software Microsoft Excel 2013 e em seguida foram calculadas as medidas de dispersão, média, mediana e desvio padrão de todas as variáveis. A seguir, para realizar a estatística, foi utilizado o programa GraphPadPrism v5.0 com nível de significância ajustado para $\alpha=0,05(p \leq 0,05)$. Para avaliar a distribuição dos dados foi utilizado o teste de Kolmogorov Smirnov, para as comparações foram utilizados o teste de Mann - Whitney para dados não paramétricos e para as correlações foi utilizado o teste de Spearman. Para melhor visualização dos resultados, estes foram apresentados em gráficos e tabelas.

\section{RESULTADOS}

Dos 30 professores universitários avaliados, dezenove (63,3\%) não apresentavam estresse e onze $(36,7)$ se apresentavam na fase de resistência, segundo o Inventário de Sintomas de Stress de Lipp (ISSL). Em relação às variáveis antropométricas treze (43,3\%) estão no peso ideal; quatorze $(46,7 \%)$ se apresentam com sobrepeso e três (10\%) estão na faixa de obesidade.

Com relação ao nível de atividade física nove professores (30\%) não praticavam nenhum tipo de atividade física moderada, vigorosa ou até mesmo caminhada. Os outros vinte e um professores (70\%) praticavam pelo menos 60 minutos de atividade física moderada ou vigorosa por semana. 


\section{TABELA 1 - CARACTERIZAÇÃO DA AMOSTRA DE PROFESSORES UNIVERSITÁRIOS POR NIVEIS DE ESTRESSE (ISSL).}

\begin{tabular}{|c|c|c|c|}
\hline \multicolumn{2}{|c|}{ Professores (ISSL = Não Apresenta) } & \multicolumn{2}{|c|}{$\begin{array}{l}\text { Professores } \\
\text { Resistência) }\end{array}$} \\
\hline $\begin{array}{l}\text { Características } \\
(n=19)\end{array}$ & $\begin{array}{l}\text { Média } \pm \text { Desvio } \\
\text { padrão }\end{array}$ & $\begin{array}{l}\text { Características } \\
(n=11)\end{array}$ & $\begin{array}{l}\text { Média } \pm \text { Desvio } \\
\text { padrão }\end{array}$ \\
\hline Idade (anos) & $41,68 \pm 9,632$ & Idade (anos) & $41,64 \pm 7,593$ \\
\hline Peso $(\mathrm{Kg})$ & $76,94 \pm 10,21$ & Peso $(\mathrm{Kg})$ & $75,35 \pm 14,71$ \\
\hline Altura (m) & $1,71 \pm 0,08777$ & Altura (m) & $1,68 \pm 0,07937$ \\
\hline IMC (Peso/Altura $\left.{ }^{2}\right)$ & $26,15 \pm 2,296$ & IMC (Peso/Altura ${ }^{2}$ ) & $26,59 \pm 4,083$ \\
\hline $\begin{array}{l}\text { Tempo de } \\
\text { Docência (Anos) }\end{array}$ & $11,53 \pm 5,891$ & $\begin{array}{l}\text { Tempo de Docência } \\
\text { (Anos) }\end{array}$ & $13,73 \pm 8,332$ \\
\hline $\begin{array}{l}\text { Atividade Física } \\
\text { Moderada ou } \\
\text { Vigorosa } \\
\text { (Minutos por } \\
\text { Semana) }\end{array}$ & $126,3 \pm 120,2$ & $\begin{array}{l}\text { Atividade Física } \\
\text { Moderada ou } \\
\text { Vigorosa } \\
\text { (Minutos por } \\
\text { Semana) }\end{array}$ & $145,5 \pm 125,8$ \\
\hline $\begin{array}{l}\text { PA Sistólica } \\
\text { (mmhg) }\end{array}$ & $121,1 \pm 12,86$ & PA Sistólica (mmhg) & $117,3 \pm 13,48$ \\
\hline $\begin{array}{l}\text { PA Diastólica } \\
\text { (mmhg) }\end{array}$ & $77,89 \pm 8,550$ & $\begin{array}{l}\text { PA Diastólica } \\
\text { (mmhg) }\end{array}$ & $76,36 \pm 6,742$ \\
\hline $\mathrm{FC}(\mathrm{bpm})$ & $74,11 \pm 12,17$ & $\mathrm{FC}(\mathrm{bpm})$ & $86,36 \pm 10,62$ \\
\hline StO2 (\%) & $96,89 \pm 0,8093$ & StO2 (\%) & $97,27 \pm 0,7862$ \\
\hline
\end{tabular}

A mediana do índice RMSSD dos professores que não apresentavam estresse foi de $22,60 \mathrm{~ms}$, e nos professores que se apresentavam em fase de resistência foi de 19,60 ms. A mediana do índice pNN50\% dos professores que não apresentavam estresse foi de $3,70 \mathrm{~ms}$, e nos professores que se apresentavam em fase de resistência foi de 2,40 ms. A mediana do índice LF dos professores que não apresentavam estresse foi de $638,0 \mathrm{~ms}$, e nos professores que se apresentavam em fase de resistência foi de 564,0 ms. A mediana do índice HF dos professores que não apresentavam estresse foi de 160,0 ms, e nos professores que se apresentavam em fase de resistência foi de 134,0 ms. 
TABELA 2 - VALORES DOS ÍNDICES DE VARIABILIDADE DA FREQUÊNCIA CARDIACA DOS PROFESSORES UNIVERSITÁRIOS POR NIVEIS DE ESTRESSE (ISSL).

\begin{tabular}{|c|c|c|c|}
\hline \multicolumn{2}{|c|}{ Professores (ISSL = Não Apresenta) } & \multicolumn{2}{|c|}{$\begin{array}{l}\text { Professores (ISSL }=\text { Fase de } \\
\text { Resistência) }\end{array}$} \\
\hline $\begin{array}{l}\text { Índices de VFC } \\
(n=19)\end{array}$ & $\begin{array}{l}\text { Média } \pm \text { Desvio } \\
\text { padräo }\end{array}$ & $\begin{array}{l}\text { Índices de VFC } \\
(n=11)\end{array}$ & $\begin{array}{l}\text { Média } \pm \text { Desvio } \\
\text { padräo }\end{array}$ \\
\hline RMSSD (ms) & $21,32 \pm 5,289$ & RMSSD (ms) & $21,55 \pm 10,90$ \\
\hline pNN50\% (\%) & $3,489 \pm 2,322$ & pNN50\% (\%) & $4,864 \pm 8,586$ \\
\hline LF (ms2) & $784,2 \pm 592,9$ & LF (ms2) & $770,6 \pm 677,8$ \\
\hline $\mathrm{HF}(\mathrm{ms} 2)$ & $155,1 \pm 72,88$ & $\mathrm{HF}(\mathrm{ms} 2)$ & $264,2 \pm 427,5$ \\
\hline
\end{tabular}

Legenda: n: populaçåo. ISSL: Inventário de Sintomas de Stress de Lipp. RMSSD: Raiz quadrada da média dos quadrados das diferencas de sucessivos intervalos R-R. pNN50: Porcentagem das diferenças sucessivas entre os intervalos RR que são maiores que $50 \mathrm{~ms}$. HF: Alta Frequência durante um intervalo de $0,15-0,40 \mathrm{~Hz}$. LF: Baixa frequência correspondente ao intervalo de $0,04 \mathrm{a} 0.15 \mathrm{~Hz}$. ms: milissegundos. $\mathrm{ms} 2$ : milissegundos ao quadrado. \%: porcentagem.

Fonte: $\mathrm{O}$ autor, 2018

Com relação aos dados da Figura 1: A, B, C e D, foi realizado a análise estatística dos dados, na qual aplicou-se o teste de Kolmogorov Smirnov, resultando-se em dados não paramétricos. Em seguida foi aplicado o teste de Mann - Whitney, na comparação dos valores médios dos índices RMSSD ( $p<$ $0,6513)$, pNN50\% ( $p<0,4012)$, LF $(p<0,7632)$ e HF $(p<0,7962)$, entre os professores universitários que não apresentavam estresse e aqueles em fase de resistência, não constatando diferença significativa.

Figura 1

A, B, C e D) COMPARAÇÃO DOS VALORES MÉdIOS DOS ÍNDICES LF, HF, RMSSD E PNN50\% ENTRE OS PROFESSORES UNIVERSITÁRIOS QUE NÃO APRESENTAVAM ESTRESSE E AQUELES EM FASE DE RESISTÊNCIA.
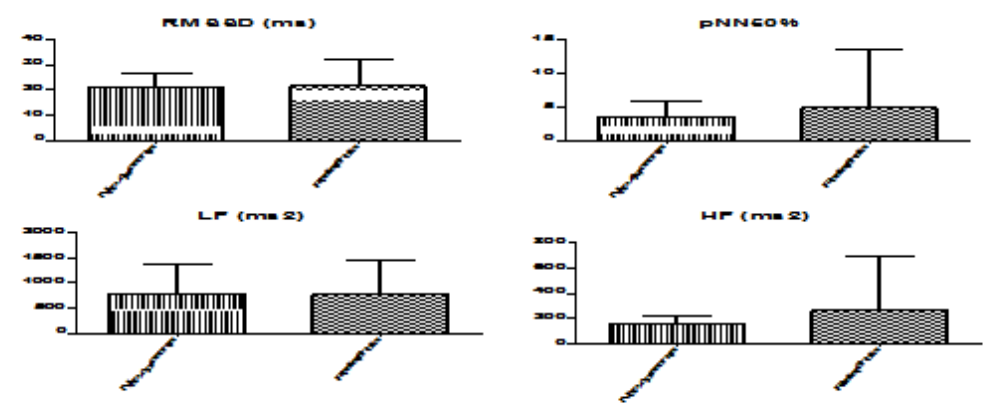

Os dados estão apresentados pela média, com valor $\mathrm{p} \leq 0,05$. Fonte: O autor (2018)

Com relação aos dados da Figura 2: A, B, C e D, foi realizado a análise estatística dos dados, na qual aplicou-se o teste de Spearman para realizar a correlação dos valores médios dos índices RMSSD $(p<0,7530)$ e $(r-0,05994)$, pNN50\% $(p<0,9108)$ e $(r-0,02135)$, LF $(p<0,4092)$ e $(r 0,1564)$ e HF $(p<0,1966)$ 
e $(r-0,2425)$ dos professores universitários e o Nível de Atividade física, Tempo

de Docência e IMC, não constatando correlação significativa.

\section{FIGURA 2:}

\section{A, B, C e D) CORRELAÇÃO ENTRE OS VALORES MÉDIOS DOS ÍNDICES RMSSD, PNN50\% LFE HF DOS PROFESSORES UNIVERSITÁRIOS E O NÍVEL DE ATIVIDADE FÍSICA, TEMPO DE DOCÊNCIA E IMC.}
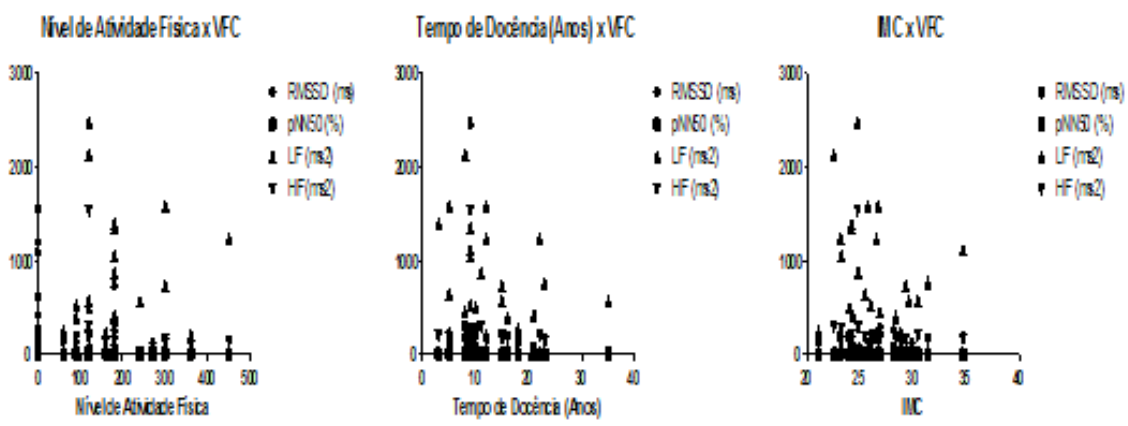

Os dados estão apresentados pela média, com valor $p \leq 0,05$. Fonte: O autor (2018).

\section{DISCUSSÃO}

O presente estudo evidenciou que os professores universitários avaliados não apresentavam estresse ou estavam na fase de resistência segundo o ISSL, ou seja, estes apresentavam baixos níveis de estresse. Estes achados corroboram com o estudo de Rosário(2014) onde também foi avaliado o nível de estresse em professores universitários através do ISSL demonstrando que mais da metade dos professores não apresentavam estresse e $90 \%$ daqueles que apresentavam, estavam na fase de resistência, o que também demonstra baixos níveis de estresse nesta população.

Sugerimos que com relação ao baixo nível de estresse encontrado nesta população, deve ser levado em conta que a população avaliada faz parte apenas de uma instituição de ensino privado, não sendo levado em conta aqueles que trabalham na rede pública e supostamente podem vivenciar situações de estresse diferentes. Além disso a população avaliada é somente de professores universitários, sendo que estes vivenciam uma realidade diferente dos professores do ensino fundamental e médio.

Quando comparado os valores médios dos índices LF, HF, RMSSD e pNN50\% entre os professores universitários que não apresentavam estresse e aqueles em fase de resistência, ficou evidenciado que não houve diferença estatística significativa, ou seja, o nível de estresse não demonstrou influência nos índices de variabilidade da frequência cardíaca (VFC) nesta população. Diante destes achados devemos ainda levar em conta que nenhum professor apresentou altos índices de estresse, fator este que poderia influenciar nos resultados, ou seja, a partir deste estudo não podemos concluir se altos índices de estresse interferem ou não nos índices de VFC.

Ainda sobre os achados do parágrafo anterior podemos verificar que baixos índices de estresse não influenciam na modulação autonômica cardíaca, visto 
que os índices de VFC se encontram preservados e compatível com os índices dos professores que não apresentavam estresse. A partir disso podemos inferir que o funcionamento do sistema nervoso autônomo da população avaliada se encontrava preservado mediante a presença de baixos níveis de estresse.

A presente pesquisa evidenciou também que não houve correlação linear estatisticamente significativa entre o nível de atividade física e os índices de VFC, porém a partir disto não podemos afirmar que não há importância clínica desta variável, visto que não foi realizado uma abordagem de análise não linear, além do fato de que quando analisados os dados individuais de cada voluntário podemos observar que os maiores índices de VFC eram daqueles com maior tempo de atividade física praticada por semana. A partir disso sugerimos a realização de novos estudos longitudinais que abordem a questão do nível de atividade física e sua influência sobre o sistema nervoso autônomo a partir da análise da VFC.

Com relação ao tempo de docência e o Índice de Massa Corporal (IMC), também não houve correlação linear estatisticamente significativa com os índices de VFC, demonstrando que estes fatores não influem sobre a modulação autonômica cardíaca. Devemos levar em conta que não foram dosados os níveis de triglicérides e colesterol, que também podem influenciar sobre estas variáveis.

A comparação dos achados do presente estudo com outras referências pertinentes na literatura se tornou limitada, visto que há uma escassez de estudos científicos que abordem a análise de variabilidade da frequência cardíaca em professores universitários, bem como sua relação com o estresse. Diante do exposto, esclareço ainda que o presente estudo apresenta algumas limitações tais como: Amostra relativamente pequena, visto que foram excluídos todos os voluntários que apresentassem qualquer patologia que pudesse influenciar na análise da VFC, além daqueles que não se dispuseram a participar da pesquisa. No presente estudo também não foi dosado o nível de cortisol sérico dos voluntários.

Sugere-se que novos estudos maiores e de longo prazo sejam realizados com o intuito de aumentar as referências e a confiabilidade dos achados deste estudo na literatura, visto que não há pesquisas semelhantes até o momento.

\section{CONCLUSÃO}

Os professores universitários avaliados apresentavam baixos níveis de estresse, sendo que alguns não apresentavam. Não houve diferente estatística significativa dos índices de VFC dos voluntários sem estresse comparados com aqueles que estavam em fase de resistência. Isto demonstra que baixos níveis de estresse não alteram a modulação autonômica cardíaca destes indivíduos, não predispondo a patologias cardiovasculares. Não houve ainda influência do nível de atividade física, IMC e tempo de docência sobre os índices de VFC.

\section{AGRADECIMENTOS}

Os autores agradecem a FAPEMIG pelo financiamento do projeto. 


\section{REFERÊNCIAS}

GOULART JUNIOR, Edward; LIPP, Marilda Emmanuel Novaes. Estresse entre professoras do ensino fundamental de escolas públicas estaduais. Psicologia em estudo, p. 847-857, 2008.

SOUSA, Abraao Vitoriano et al. ESTRESSE E A PROFISSÃO DOCENTE: CONCEITOS E SINTOMAS. Revista FAMA de Educação, Tecnologia e Informação, v. 1, n. 2, p. 12-16, 2016.

MARTINS, Cláudia Cristiane Filgueira; TOURINHO, Francis; SANTOS, Viviane. Estresse-normal ou patológico?. Saúde \& Transformação Social/Health \& Social Change, v. 7, n. 1, p. 001-008, 2016.

SAÚDEBUSINESS. OMS prevê que estresse e depressão vão liderar morte no mundo. 2013. Disponível em: http://www.saúdebusiness.com/notícias. Acesso em: 31 jan. 2017.

SERVILHA, Emilse Aparecida Merlin. Estresse em professores universitários na área de fonoaudiologia. Revista de Ciências Médicas, v. 14, n. 1, 2012.

FERREIRA, Ana Rita Pereira et al. Stress ocupacional e burnout em professores universitários. 2016.

RODRIGUES, Pedro Henrique et al. A influência dos fatores de risco para doenças cardiovasculares sobre a modulação autonômica cardíaca. Revista de Atenção à Saúde (antiga Rev. Bras. Ciên. Saúde), v. 14, n. 49, p. 34-40, 2016.

OLIVEIRA-SILVA, Iransé et al. VARIABILIDADE DA FREQUÊNCIA CARDÍACA, IMC E ESTRESS PRÉ-COMPETITIVO EM ATLETAS DE NATAÇÃO. REVISTA CEREUS, v. 8, n. 2, p. 100-111, 2016.

LONGHI, Allan. Variabilidade da frequência cardíaca, estresse, ansiedade e depressão em intensivistas: um estudo nas unidades de terapia intensiva da cidade de Dourados, MS. 2009.

MARÃES, V. R. F. S. Frequência cardíaca e sua variabilidade: análises e aplicações. Revista andaluza de Medicina del Deporte, v. 3, n. 1, 2010.

CAMM A, MALIK M, BIGGER J, BREITHARDT G, CERUTTI S, COHEN R, \& LOMBARDI F. Heart rate variability: standards of measurement, physiological interpretation and clinical use. Task Force of the European Society of Cardiology and the North American Society of Pacing and Electrophysiology. Circulation, v. 93, n. 5, p. 1043-1065, 1996.

ROSÁRIO, Nacha Samadi Andrade. Relação entre o condicionamento cardiorrespiratório e o nível de estresse em professores universitários. 2014.

Recebido em: 10-05-2018

Aceito em: 03-10-2019 